\title{
Multivariate spectral analysis for identifying the brain activations during olfactory perception.
}

\author{
Eleni Kroupi ${ }^{1}$, Ashkan Yazdani ${ }^{1}$, Jean-Marc Vesin ${ }^{2}$ and Touradj Ebrahimi ${ }^{1}$
}

\begin{abstract}
Olfactory perception is a complex phenomenon associated with other processes such as cognition and emotion. Due to this complexity, there are still open issues and challenges regarding olfactory psychophysiology. One challenge concerns the investigation of the hedonic dimension of olfaction, and how it affects the power of the brain oscillations. Although there are some EEG studies exploring the changes in the power of the brain oscillations during olfactory perception, they use simple power spectral analysis techniques and vary much in terms of the reported findings. To reduce this variability, we propose the use of multivariate spectral analysis, to reveal only the frequency patterns of the EEG signals that contribute the most to olfactory perception. The goal is to investigate how these frequency patterns are affected by hedonically different odors throughout the cortex.
\end{abstract}

\section{INTRODUCTION}

Olfactory sense is one of the most important senses of many animals. Benefiting from the ability to perceive and recognize different smells, animals can interact better with their surroundings through identifying food, mates, and prays. Using their olfaction, animals can also detect the presence of predators and escape from various dangers. Similarly for humans, olfaction has a significant importance and can modulate behavioral and social reactions. Odor stimuli can elicit emotions and have a general impact on mood and associated behavior.

Odor stimuli are created by mixtures of volatilized chemicals and are directly perceived by the human olfactory bulb through the olfactory epithelium. The information flows from the olfactory bulb to highly synthetic cortical areas, such as the piriform cortex [1]. This cortical region has a unique architecture and a unique contribution to odor encoding and perception, so it has been extensively studied [2]. Regarding the processes inside the olfactory bulb, odor-related activity shows an oscillatory nature [3]. These oscillations result from local field potentials and can be categorized into four different frequency bands, namely theta (4-7 Hz), alpha (8-13 Hz), beta (14-29) and gamma (30-100 Hz). Several studies have tried to capture the oscillatory nature of the olfactory perception from electroencephalographic (EEG) signals. However, there is much variability in terms of the reported findings. For instance, differences in the amount of alpha activity and an increase of theta activity were observed when subjects were exposed to fragrances and the EEG was recorded from 4 electrodes [4]. However, other authors

\footnotetext{
${ }^{1}$ Multimedia Signal Processing Group (MMSPG), École Polytechnique Fédérale de Lausanne (EPFL)

${ }^{2}$ Applied Signal Processing Group (ASPG), École Polytechnique Fédérale de Lausanne (EPFL)
}

suggested that there are no changes in alpha activity but there are increases in theta activity [5]. Moreover, in a more recent study [6] a decrease in the activity of theta and alpha bands was found. In the same study, an increase in the high frequency EEG components $(11-25 \mathrm{~Hz})$ was reported.

One possible reason for the variability among EEG olfactory studies is that in addition to the direct activation of the olfactory bulb and the piriform cortex, the human olfactory path is interconnected with other cortical and limbic structures, such as the amygdala [1]. The role of the amygdala in emotion, memory, and autonomic control directly associates olfaction to these processes and adds complexity to the odor perceptual experience. Due to this complexity, there are still open issues and more research needs to be conducted on olfactory perception experience.

One approach is to investigate the affective dimension of olfactory perception. In affect recognition research, several different audio/visual stimuli are presented to subjects while their brain activity is being recorded. The subjects are asked to provide their self-assessed induced emotions (in terms of pleasantness and excitation), and such data is used to determine the changes in the brain activity during perception of different emotions. The same approach can be used to understand the affective dimension of olfaction. More specifically, similarly to audio/visual stimuli, odors can be classified in terms of two different aspects. Odor intensity, which is perceived as the strength of an odor and can range from no odor to intolerable, and odor pleasantness which can take values from extremely unpleasant to extremely pleasant. Thus, subjects will be asked to smell different odors and provide their self-assessed perception in terms of intensity and pleasantness, in order to investigate the effect of odor perception on brain activity.

To this end, in the current study we are interested in investigating the oscillatory effect of differences between a very pleasant odor and a very unpleasant one in various brain regions. In order to avoid the loss of important information that often arises from the improper statistical integration of the features from many electrodes, we propose to use multivariate spectral analysis. More specifically, we use the spectral envelope [7] which reveals the common frequency patterns of the signals under investigation. By using only the common frequency patterns of the EEG signals, which contribute the most to olfactory perception, we avoid dealing with the parts of the EEG power spectrum that do not contain much useful olfactory information and might also lead to variability among EEG olfactory studies.

This paper is organized as follows. Section II describes 


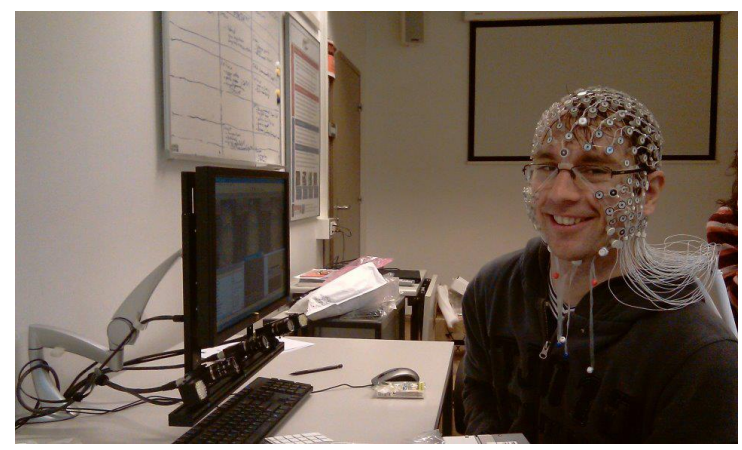

Fig. 1. A participant shortly before the beginning of the experiment.

the experimental protocol and the methods used in this study. The results and the further discussion are detailed in Section III. Finally, the conclusions are presented in Section IV.

\section{MATERIALS AND METHODS}

\section{A. Participants}

Five right-handed male $\mathrm{PhD}$ students and Postdoctoral researchers participated in the study. They were between 26 and 32 years old and were recruited from École Polytechnique Fédérale de Lausanne (EPFL). According to their self-reports, none of them had a history of injury in the olfactory bulb or incapability of smelling. All of them stated that they were not suffering from any respiratory, mental or chronic disease. Before the beginning of the experiments, participants filled in an informed consent form. During the experiments, participants were seated on a comfortable chair with controlled environment.

\section{B. Set up}

The EEG was recorded at $250 \mathrm{~Hz}$ sampling rate from 256 electrodes placed at the standard EGI positions on the scalp. An EGI's Geodesic EEG System (GES) 300 was used to record, amplify, and digitalize the EEG signals. Figure 1 illustrates the EEG electrodes and a subject before the beginning of the experiment.

\section{Experimental protocol}

Subjects were asked to close their eyes and breath normally while the experimenter was moving bottles with different odorants towards them to smell. The odorants consisted of valerian, lotus flower, rosewater and fermented Swiss Tomme goat cheese.

The experiment consisted of four runs. During each run, after a "smell" command the experimenter was randomly choosing a bottle with an odor to place it under the subject's nose $(1-2 \mathrm{~cm}$ under both nostrils) and keep it there for about 2 seconds, constituting a single trial. This process was repeated for twenty to thirty times with the same odor resulting in twenty to thirty single trials. The number of single trials was selected so as to gather enough data.

The time between two single trials of the same odor was set to four seconds in order to avoid adaptation and subject's fatigue. After one run was over, the subjects were given two

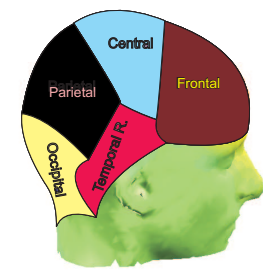

(a) Headplot

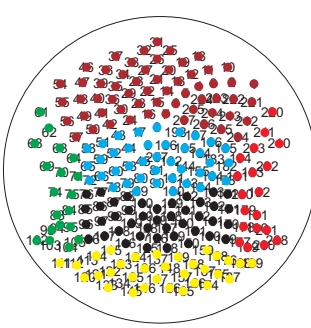

(b) Topoplot.
Fig. 2. Separation into six brain regions (frontal, central, left temporal, right temporal, parietal and occipital).

minutes break in order to forget the odor (to avoid masking effect) and in order for the odor to be evacuated. During this break, the subjects were asked to rate the odor in terms of pleasantness, in a scale from 0-10, ranging from extremely unpleasant to extremely pleasant. After this break another odor was randomly selected and the same procedure was repeated until all four odors were presented. In this study, only the most pleasant and the most unpleasant odor for each participant were used for data analysis.

\section{Signal processing}

EEG electrodes in which muscle activity was discernible were rejected manually, leading to a total 216 electrodes for processing and analysis. EEG signals were referenced to the $\mathrm{Cz}$ electrode and re-referenced to the common average. Single trials were generated so as to last for one second after the stimulus onset. Furthermore, in order to remove the influence of stimulus-unrelated variations, a one-second baseline was recorded before each run.

\section{E. Feature extraction and classification}

The electrodes were manually clustered, according to their position on the head, into six different categories, namely frontal, central, left temporal, right temporal, parietal and occipital (Figure 2). Then, multivariate spectral analysis was performed using the spectral envelope [7]. The spectral envelope systematically selects the spectral components that appear simultaneously in different signals, so it enhances the periodic signatures that are common for all signals, and attenuates the noise. The algorithm can be described as follows; let $\mathbf{x}(t), t=0, \pm 1, \pm 2, \ldots$, be a time series in $\Re^{m}$. If $g(t, \boldsymbol{\beta})=\boldsymbol{\beta}^{H} \mathbf{x}(t)$, where $\boldsymbol{\beta}$ is a $m \times 1$ real or complex column vector and $H$ the conjugate transpose operator, then the spectral envelope of $\mathbf{x}(t)$ is defined as

$$
\lambda(\omega)=\sup \left\{\frac{P_{g}(\omega, \boldsymbol{\beta})}{\sigma_{g}^{2}(\boldsymbol{\beta})}\right\} \quad-\pi<\omega \leq \pi,
$$

where $P_{g}(\omega, \boldsymbol{\beta})$ is the power spectral density (PSD) of $g(t, \boldsymbol{\beta})$ and $\sigma_{g}^{2}(\boldsymbol{\beta})$ its variance [7]. In this study, the PSD was estimated using Welch's non-parametric method with windows of 128 samples (to make plain use of the FFT) and $80 \%$ overlapping. The spectral envelope was estimated for both the single trials and the baselines. The baseline spectral envelope was then subtracted from the single trial spectral envelope, in order to extract an estimate of the distance 


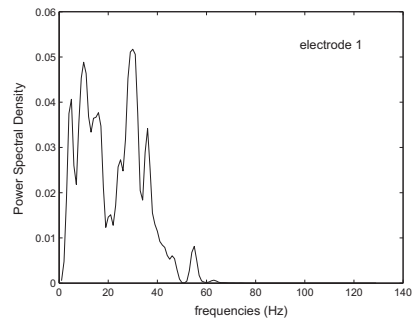

(a) PSD of electrode 1 .

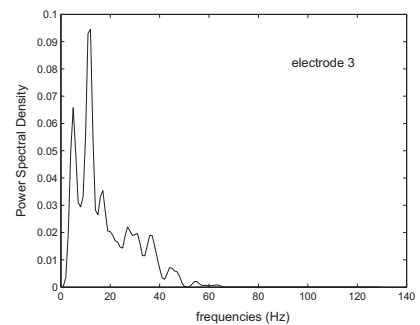

(c) PSD of electrode 3 .

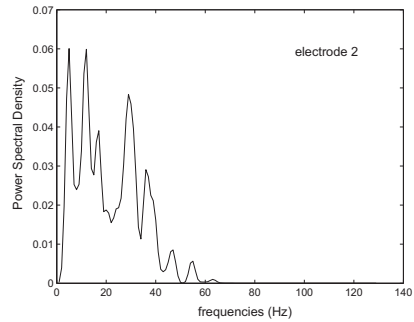

(b) PSD of electrode 2 .

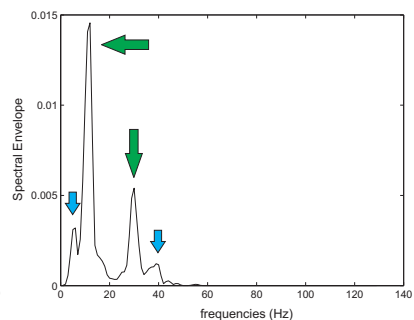

(d) Spectral envelope.
Fig. 3. This figure represents an example of the spectral envelope for three frontal electrodes. Figures 3(a)-3(c) show the PSD of the three frontal electrodes, whereas Figure 3(d) shows the spectral envelope of these electrodes.

between the envelope of the single trials and the envelope of the pre-stimulus period. The spectral envelope was used in order to detect and emphasize the common components of the PSD of electrodes that belong to the same brain region. Figure 3(d) shows the spectral envelope of three frontal electrodes whose PSD, estimated using Welch's method, is presented in Figures 3(a)-3(c). The enhanced common frequency components of the three signals are pointed by arrows. The spectral envelopes of the electrodes that belong to the same brain region were computed for all six regions, resulting in six different envelopes for each subject. For this work, the changes in the power of different frequency bands of the spectral envelopes were studied. These frequency bands consisted of theta band (4-7 Hz), alpha band (8-13 $\mathrm{Hz}$ ), low beta band (14-16 Hz), middle beta band (16-20 $\mathrm{Hz}$ ), high beta band (21-29 Hz) and gamma band (30-47 $\mathrm{Hz}$ ).

The Hilbert-Schmidt independence criterion (HSIC) [8] was used in order to investigate the dependence between the pleasant and the unpleasant odors across the 5 subjects, for each frequency band and for each brain region. The HSIC test was selected because it outperforms traditional correlation tests and because it considers the subjects as independent and identically distributed samples. The HSIC test uses a kernel independence measure in order to detect linear or non-linear dependencies between arbitrary input variables. In contrast with other statistical tests, the HSIC test either rejects or accepts the null hypothesis, depending on a certain threshold [8]. Following [8], the null hypothesis was that the pleasant and the unpleasant odors are independent. The $p$-value of the HSIC test was computed using Bootstrap approximation with $10^{4}$ re-sampling repetitions and the confidence interval was set to $95 \%$ to avoid Type II errors in which a false hypothesis
TABLE I

SIGNIFICANT DIFFERENCES FOR FREQUENCY BANDS AND REGIONS

\begin{tabular}{lcccccc}
\hline $\begin{array}{l}\text { Bands/ } \\
\text { Regions }\end{array}$ & F & C & L.T & R.T & P & O \\
\hline Theta & SI & SI & NS & NS & SI & NS \\
Alpha & NS & NS & NS & NS & NS & SI \\
Low & SI & SI & NS & SI & SI & NS \\
Beta & & & & & & \\
Beta & NS & NS & NS & SI & SI & SI \\
High & SI & SI & SI & NS & NS & NS \\
Beta & & & & & & \\
Gamma & SI & SI & SI & SI & NS & NS \\
\hline
\end{tabular}

might not be rejected. Finally, the kernel size of the HSIC test was set to the median distance between the points [8].

A Support Vector Machine (SVM) classifier with radial basis function (RBF) kernel was used to classify between the EEG signals that correspond to pleasant and unpleasant odors for each brain region. The RBF kernel was used due to the fact that the number of features was not very large and that this kernel considers the possible non-linear relationships between class labels and features. The LIBSVM package was used for this study [9]. The parameters of the classifier were optimized for each brain region, using a grid search in a leave-one-out cross-validation scheme [9].

\section{RESULTS AND DISCUSSION}

The results of the HSIC test are presented in Table I. SI indicates Significant Independence, whereas NS indicates No Significant independence between the pleasant and the unpleasant odors. The abbreviations F, C, L.T, R.T, P and O stand for Frontal, Central, Left Temporal, Right Temporal, Parietal and Occipital, respectively (Figure 2). According to Table I, different frequency bands seem to contain hedonic olfactory information for different brain regions. For instance, theta, low and high beta, and gamma bands contain hedonic olfactory information reflected in the frontal, central and temporal regions. It has been established that beta and gamma bands are highly associated with cognition [10], [11], and that theta band is associated with attentional demands and working load [12]. Moreover, beta band also reflects emotional characteristics [10]. Hence, cognitive and emotional processes seem to be reflected in the frontal, central and temporal lobes during hedonic olfaction. This finding corroborates the studies in affect research, in which the frontal lobe is shown to be associated with pleasant and unpleasant emotions [13]. Moreover, the above finding confirms the role of the piriform cortex in olfaction, which lies at the junction of the frontal and the temporal lobes [2].

Figures 4(a)-4(b) show the brain activations that occur in different frequency bands of the spectral envelope during perception of pleasant and unpleasant odors versus a neutral baseline. According to these figures, the power corresponding to theta, alpha, and high beta bands of the frontal and central lobes decreases while perceiving pleasant odors, while the power corresponding to gamma band slightly increases. Increased frontal gamma activity can be interpreted as re- 


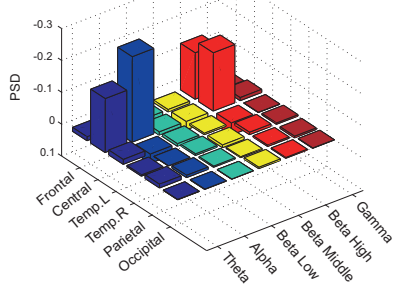

(a) Pleasant odors.

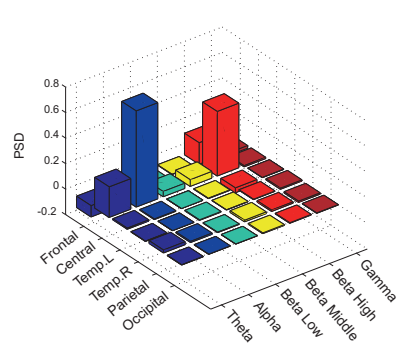

(c) Unpleasant versus pleasant odor.

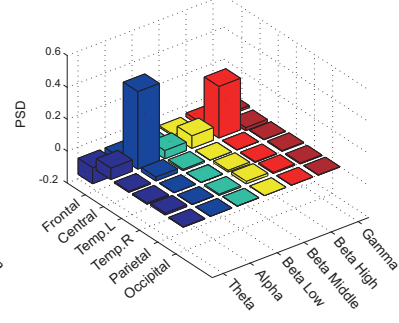

(b) Unpleasant odors.

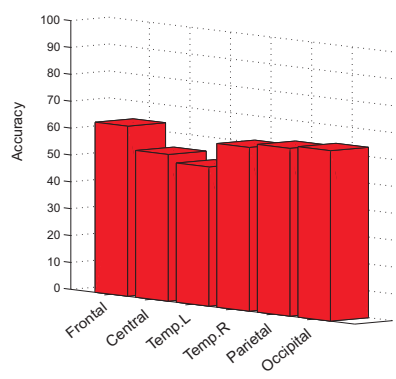

(d) Classification accuracy.
Fig. 4. Brain-region activations from pleasant and unpleasant odors. Figure 4(a) shows the behavior of the frequency bands during perceiving pleasant odors while Figure 4(b) during perceiving unpleasant odors. Figure 4(c) shows the difference in the power of the frequency bands of the pleasant subtracted from the power of the unpleasant odors. Finally, 4(d) shows the classification accuracy for each brain region.

flecting the synchronization of neural activity in the frontal lobe, and is associated with processes such as alertness, attentiveness and memory [14]. Also, theta and alpha bands are indicators of attention and working memory load [10], [12]. Thus, olfaction seems to be highly associated with cognitive processes. Furthermore, in Figure 4(c), the frontal and central high beta power changes with an unpleasant odor versus a pleasant one. This verifies the affective dimension of olfaction, where beta power is associated with positive and negative emotions [10].

In order to investigate which brain regions are mainly responsible for the separability between a pleasant and an unpleasant odor in terms of all bands, an SVM classifier was used with leave-one-subject-out cross validation. More precisely, the classifier was trained using the band powers of the spectral envelopes acquired from four subjects and was tested on the single trials of the remaining subject. This was repeated until each subject was considered once as a test subject. The single trial classification results for each brain region are presented in Figure 4(d).

As expected, Figure 4(d) shows higher classification accuracy in the frontal region (63.4\%), indicating that this region is the most active when perceiving hedonically different odors. As explained before, this finding corroborates the role of piriform cortex in olfaction, as well as most of the affective studies that associate the frontal lobe activation with positive and negative emotions. However, high classification accuracy is also obtained in the occipital part of the brain $(63.3 \%)$, although subjects were with eyes closed. Similar to Cherninskii et al. [6], this may reflect the creation of mental visual images in the perception of odors. Finally, high classification accuracy in the frontal and the occipital regions indicates that EEG signals from these brain regions can be used for the discrimination and automatic recognition among hedonically different odors.

\section{CONCLUSIONS}

In this study we explored the affective dimension of olfaction through the analysis of the effects of pleasant and unpleasant odors in different brain regions. We used the spectral envelope to capture the EEG power changes across different brain bands and brain regions, by enhancing the most interesting common parts of the power spectral density of the EEG signals. This study revealed that olfactory perception of hedonically different odors involves underlying processes of attention, memory and emotion, which are depicted in alpha, theta, beta and gamma oscillations mainly of the frontal and occipital lobes.

\section{ACKNOWLEDGMENT}

The research leading to these results has been performed in the frameworks of Swiss National Foundation for Scientific Research (FN 200020-132673-1), and the NCCR Interactive Multimodal Information Management (IM2). Furthermore, the authors would like to thank all subjects, who kindly participated in the experiments.

\section{REFERENCES}

[1] D. Wilson and R. Stevenson, "Learning to smell," Baltimore: John Hopkins UP, 2006.

[2] M. Bensafi, "The role of the piriform cortex in human olfactory perception: Insights from functional neuroimaging studies," Chemosensory Perception, pp. 1-7, 2011.

[3] E. Adrian, "The electrical activity of the mammalian olfactory bulb," Electroencephalography and clinical Neurophysiology, vol. 2, no. 1-4, pp. 377-388, 1950.

[4] T. Lorig and G. Schwartz, "EEG activity during relaxation and food imagery." Imagination, Cognition and Personality, 1988.

[5] W. Klemm, S. Lutes, D. Hendrix, and S. Warrenburg, "Topographical EEG maps of human responses to odors," Chemical senses, vol. 17, no. 3, p. 347, 1992.

[6] A. Cherninskii, I. Zima, N. Makarchouk, N. Piskorskaya, and S. Kryzhanovskii, "Modifications of EEG related to directed perception and analysis of olfactory information in humans," Neurophysiology, vol. 41, no. 1, pp. 63-70, 2009.

[7] A. McDougall, D. Stoffer, and D. Tyler, "Optimal transformations and the spectral envelope for real-valued time series," Journal of statistical planning and inference, vol. 57, no. 2, pp. 195-214, 1997.

[8] A. Gretton, K. Fukumizu, C. Teo, L. Song, B. Schölkopf, and A. Smola, "A kernel statistical test of independence," 2008.

[9] C. Chang and C. Lin, "LIBSVM: a library for support vector machines," Software available at http://www.csie.ntu.edu.tw/ cjlin/ libsvm/, 2001.

[10] W. Ray and H. Cole, "EEG alpha activity reflects attentional demands, and beta activity reflects emotional and cognitive processes," Science, vol. 228 , no. 4700 , p. $750,1985$.

[11] R. Llinas and U. Ribary, "Coherent $40-\mathrm{Hz}$ oscillation characterizes dream state in humans," Proceedings of the National Academy of Sciences, vol. 90, no. 5, p. 2078, 1993.

[12] A. Gevins, M. Smith, L. McEvoy, and D. Yu, "High-resolution EEG mapping of cortical activation related to working memory: effects of task difficulty, type of processing, and practice." Cerebral Cortex, vol. 7, no. 4, pp. 374-385, 1997.

[13] R. Davidson, "Affective neuroscience and psychophysiology: Toward a synthesis," Psychophysiology, vol. 40, no. 5, pp. 655-665, 2003.

[14] J. Kaiser and W. Lutzenberger, "Induced gamma-band activity and human brain function," The Neuroscientist, vol. 9, no. 6, p. 475, 2003. 